\title{
Rearview Mirror Reflectivity and the Quality of
}

Distance Information Available to Drivers

\author{
Michael J. Flannagan \\ Michael Sivak \\ Dennis S. Battle \\ Takashi Sato \\ Eric C. Traube
}

The University of Michigan

Transportation Research Institute

Ann Arbor, Michigan 48109-2150

U.S.A.

Report No. UMTRI-92-46

December 1992 
Technical Report Documentation Page

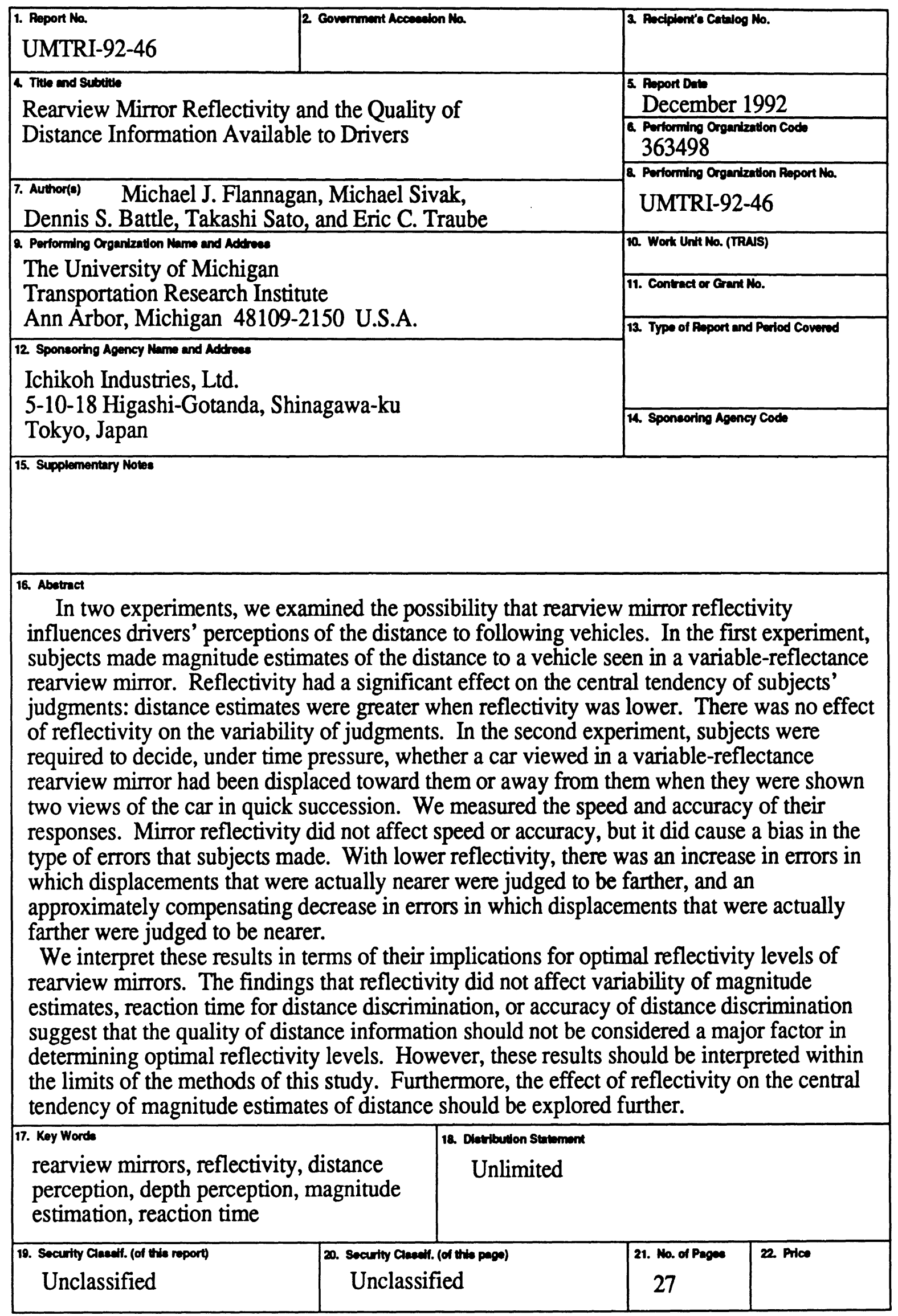




\section{Acknowledgements}

We thank Ichikoh Industries, Ltd. for its generous support of this research. 


\section{Contents}

Acknowledgements...............................................................ii

Introduction........................................................................

\section{Experiment 1}

Magnitude Estimation of Distance ..........................................2

Experiment 2

Distance Discrimination ...................................................13

General Discussion ............................................................23

References ..................................................................24 


\section{Introduction}

In the past, the problem of headlamp glare reflected from rearview mirrors has been addressed primarily by using two-position, prism mirrors. These mirrors have two reflective surfaces, with reflectivities of about 80 percent and 4 percent. The low-reflectivity surface provides adequate protection from glare in most situations (thus increasing the visibility of forward stimuli, as well as driver comfort), but it provides reduced visibility of stimuli seen in the mirror. It is difficult to define exactly what a driver needs to see in a rearview mirror, and just how well he or she needs to see it, but it is possible that 4-percent reflectivity is lower than optimal in many driving situations. Many drivers believe it to be too low. In a recent survey, 33 percent of respondents reported reducing or forgoing the use of the antiglare setting because of inability to see to the rear (Flannagan \& Sivak, 1990). Also, several studies, based on a variety of methods, have recommended reflectivity levels higher than 4 percent as compromises between visibility and glare protection (Mansour, 1971; Olson, Jorgeson, \& Mortimer, 1974; Ueno \& Otsuka, 1988).

In recent years a variety of new technologies, including electrochromic mirrors (e.g., Lynam, 1987), have become available that may provide a better solution to the problem of glare from rearview mirrors. Unlike prism mirrors, which provide two discrete reflectivity levels, these mirrors provide continuously variable reflectivity. Therefore they may be able to achieve a better tradeoff between glare protection and visibility to the rear. This new capability has provided increased motivation to understand rearward vision requirements.

Two special circumstances influence rear vision requirements at night. First, in principle, the only information a driver needs from the rear concerns moving vehicles. All other objects, including, for example, pedestrians and parked vehicles, are not in danger of being hit once they are abreast of, or behind, the driver's own vehicle. Second, moving vehicles are always marked by headlamps and marker lamps. These facts can be used to argue that mirror reflectivity should have little effect on important aspects of rearward vision at night; even at very low reflectivity, certainly below 4 percent, headlamps themselves are luminous enough to be detected easily.

However, there are at least two general ways in which this analysis may be incomplete. First, there may be stimuli other than lamps that are important for a driver to detect. A vehicle in an adjacent lane may be positioned so that its headlamps are in a driver's blind spot, in front of the fields of view of the rearview mirrors, while the rear of the vehicle extends backward into the mirror fields of view. This situation may be common in dense traffic on multilane highways, an environment in which rearview mirrors are important for changing lanes. In such cases the ability to see the unlighted side aspect of a vehicle in an adjacent lane may be 
useful to a driver. The ability to see empty pavement as a positive indication that no vehicle is present may also be useful, or at least drivers may believe it to be useful. Second, the above analysis may be incomplete in that it considers only the basic detectability of visual information, rather than its quality or salience. Even if headlamps of following vehicles are detected perfectly in a rearview mirror with 4 percent reflectivity, the ability of drivers to make fast and accurate judgments about the locations of vehicles behind them may be diminished.

The present study addressed this second possibility. We quantified the effects of mirror reflectivity on both the speed and accuracy of subjects' distance judgments. We were interested in two potentially independent ways in which reflectivity might affect accuracy: changes in constant error (e.g., lower reflectivity might cause stimuli to be perceived as farther away) and changes in variable error (e.g., lower reflectivity might make perceptions less consistent). Either type of effect could occur alone or in combination with the other.

We conducted two experiments, involving different distance-judgment tasks that provided complementary types of information about the accuracy of subjects' perceptions of distance. In the first experiment, we used the psychophysical technique of magnitude estimation (Marks, 1974; Stevens \& Galanter, 1957). Subjects made magnitude estimates of the distance to a vehicle seen through a rearview mirror, while the reflectivity of the mirror and the actual distance to the vehicle were varied. Data from that task allowed us to assess directly any effects of reflectivity on both constant error and variable error, by examining the central tendency and variability of subjects' responses to repeated presentations of the same stimulus combinations. In the second experiment, subjects were required to decide, under time pressure, whether the distance to a vehicle seen through a rearview mirror had been reduced or increased. Reaction time data from that task allowed us to measure the effect of reflectivity on the speed with which subjects can make distance judgments. Error rates provided a second form of information about the variable error of distance perceptions. Any increases in variable error should be detectable by their negative effects on discrimination performance.

\section{Experiment 1: Magnitude Estimation of Distance}

\section{Method}

Subjects. Twenty licensed drivers, 10 in a younger group and 10 in an older group, were paid to participate. The younger group ranged in age from 17 to 30 , with a mean age of 24.1; the older group ranged from 61 to 73, with a mean age of 66.9. The younger group consisted of five males and five females; the older group consisted of six males and four females. 
Apparatus. Two vehicles were used in the study. A 1982 Ford Country Squire station wagon served as the observer vehicle, and was equipped with an electrochromic interior rearview mirror mounted in the standard position. The electrochromic mirror was attached to a voltage supply that could be quickly switched among preset voltage levels in order to provide different levels of reflectivity. The driver-side exterior mirror was turned far out of position so that it could not be used to view the rearward scene. The second vehicle was a 1991 Ford Taurus station wagon that was used as the stimulus vehicle. The headlamps were $15 \mathrm{~cm}$ high and $23 \mathrm{~cm}$ wide, and were separated by $122 \mathrm{~cm}$, center to center. The centers of the lamps were $68 \mathrm{~cm}$ above the pavement. Only low beams were used in this study.

Field setup. Testing was conducted in a parking lot adjoining the UMTRI building. The area was dark, with no artificial lighting in or near the lot. The setup is shown schematically in Figure 1. The observer vehicle was placed so that the rearview mirror view would be across the lot toward a set of six positions, all in a line directly behind the observer vehicle, at which the stimulus vehicle would be placed. The pavement was dry asphalt.

Stimuli. On each trial, the subject was presented with the stimulus vehicle visible in the rearview mirror at one of six distances $(30,33,36,39,42$, or $45 \mathrm{~m}$, measured between the centers of the front wheels of the vehicles), with the mirror set at one of three reflectivities $(0.06,0.22$, or 0.80 , which are separated by logarithmically equal steps). The stimulus vehicle was always presented with its low-beam headlamps on.

Photometry. Because subjects' eye positions were not strictly controlled, it was not practical to measure illumination by the headlamps of the stimulus vehicle at those positions directly. Instead, illumination was measured at the center of the mirror. From these measurements, taking into account mirror reflectivity and the slightly longer path length to the subjects' eyes, approximate illumination of the subjects' eye positions can be calculated. The distance from the measuring point to the stimulus-vehicle headlamps was about $0.5 \mathrm{~m}$ shorter than the optical path from the subject's eye point, through the mirror, to the headlamps. Application of the inverse-square law predicts that this difference in path length (ignoring for the moment the influence of the reflectivity of the mirror) should result in illuminance values at the eye that are only slightly lower, by about 2 or 3 percent, depending on the position of the stimulus vehicle. Because the experiment and the photometry were done with the tailgate open, rear-window glass was not a factor. $\log _{10}$ lux measured at the center of the mirror is shown in Figure 2 for each of the six positions of the stimulus vehicle. Note that illuminance was highest at the 33-m position. The pattern is consistent with expectations based on a tradeoff between the inverse-square law and the fact that at shorter distances the rearview mirror was at a higher (and, within this range, less intense) angular location in the beam patterns of the stimulus-vehicle headlamps. 


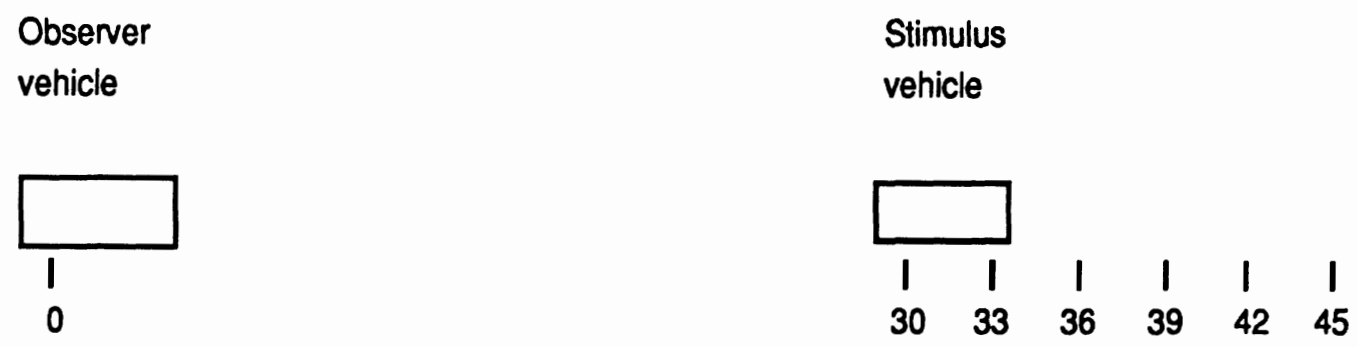

Figure 1. Plan view of the field setup for Experiment 1, showing the observer vehicle at its fixed position (front wheel centered at $0 \mathrm{~m}$ ) and the stimulus vehicle at the closest of the six positions at which it appeared (front wheel centered at $30 \mathrm{~m}$ ).

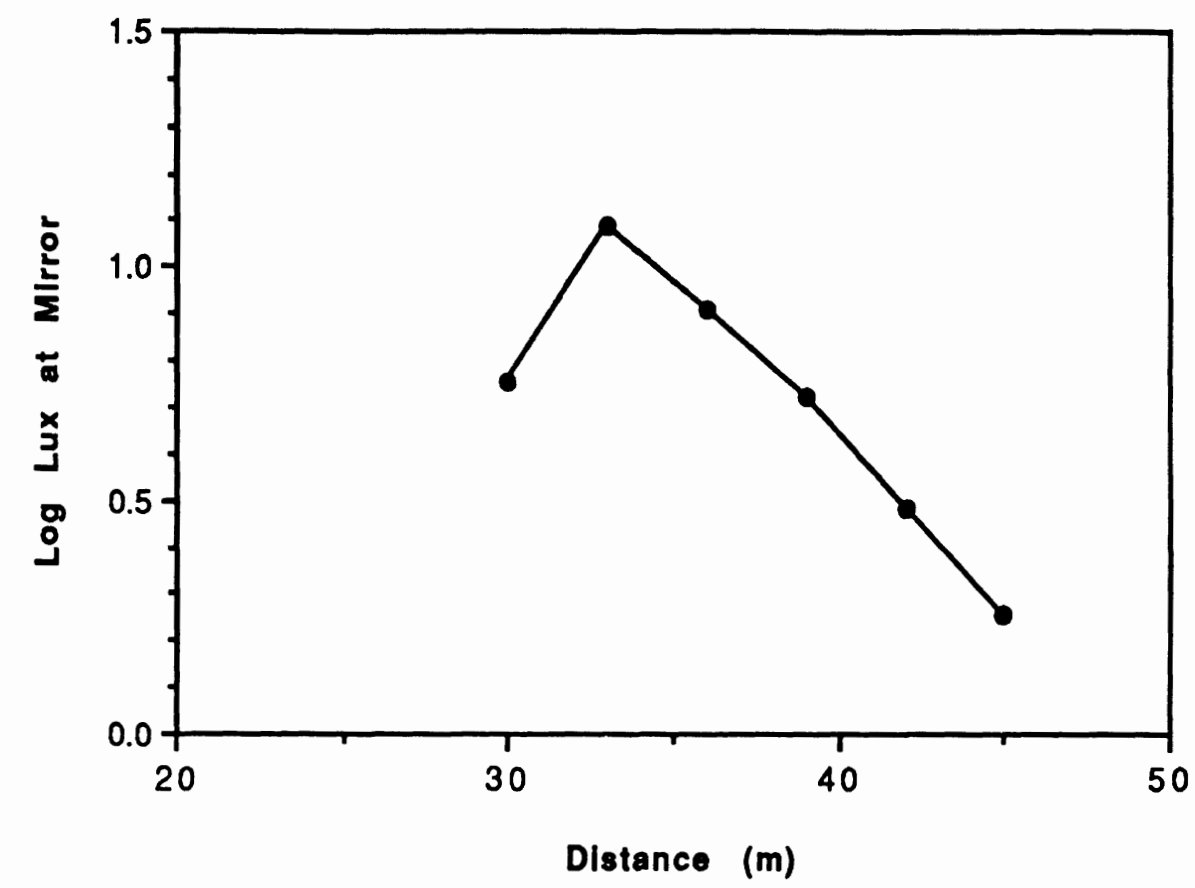

Figure 2. $\log _{10}$ lux at the rearview mirror with the stimulus vehicle at each of the distances used in Experiment 1.

Design. There were two between-subjects variables: age and sex; and three withinsubject variables: stimulus-vehicle distance, mirror reflectivity, and time (represented by dividing a session into quarters). Each subject served in a single session. Each of the 18 factorial combinations of vehicle distance (6 levels) and mirror reflectivity (3 levels) was presented four times, once during each of four blocks of trials that made up the session. Within each block the order of treatments was randomized.

Procedure. Data were collected in the early hours of the night, between approximately one half hour after sunset and midnight. Subjects were run individually. They were seated in the driver's seat of the observer vehicle and given instructions about the magnitude estimation 
task. They individually adjusted the inside rearview mirror so that the image of the stimulus vehicle at the 36-m position was centered vertically and horizontally. That provided them with a view of the pavement from the front of the stimulus vehicle to a position about $12 \mathrm{~m}$ from the rear of the observer vehicle. The tailgate of the observer vehicle was open so that the factor limiting the nearest visible point on the pavement was the field of view of the rearview mirror, not the frame of the rear window, as is usually the case.

Just before data collection began, the subjects were shown the stimulus vehicle at the 36-m position, and told to use that position as an anchor for their magnitude estimates, assigning it the value 100 . They were encouraged not to estimate distance in feet, meters, or any other conventional unit; but simply to choose numbers that represented their perception of distance relative to the anchor position. They were not told the actual distances of the anchor position or any other position at which the stimulus vehicle would be placed, nor were they told at how many different positions the vehicle would be placed.

One experimenter sat in the back seat of the observer vehicle, out of either direct or mirror view of the subject. A second experimenter drove the stimulus vehicle, moving it the short distances between the designated stimulus positions.

Just before each trial, while the subject looked down at the instrument panel of the observer vehicle, the second experimenter placed the stimulus vehicle at one of the six positions. Then, at the instruction of the first experimenter, the subject looked into the interior rearview mirror and made a numerical magnitude estimate of the distance to the stimulus vehicle. The subject then looked down at the instrument panel while the stimulus vehicle was moved for the next trial. Immediately after the subject gave the estimate, the first experimenter recorded it; and, unless mirror reflectivity was to be the same on the next trial, set the voltage to the rearview mirror so that its reflectivity would have time to stabilize before the next trial. (Because mirror reflectivity—along with stimulus-vehicle distance—was varied randomly from trial to trial, on about one third of the trials mirror reflectivity was not changed from the previous trial.) The intertrial interval was approximately $25 \mathrm{~s}$.

\section{Results and Discussion}

We assessed possible effects of mirror reflectivity and the other variables on two aspects of subjects' performance: the central tendency and the variability of their magnitude estimates. We will discuss central tendency effects first.

Effects on central tendency of estimates. We performed an analysis of variance on magnitude estimates, with age and sex as between-subjects factors; and block, distance, and mirror reflectivity as within-subject factors. This analysis yielded two significant main effects, 
and five significant two-way interactions. The two main effects are of most interest, and will be discussed first.

The effect of actual distance on magnitude estimates of distance, $F(5,80)=77.87$, $p<.0001$, is shown in Figure 3. The effect is approximately linear, and the best straight-line fit to the data in Figure 3 yields a slope of 2.83 units of averaged magnitude estimates per meter of actual distance.

Mirror reflectivity also had a significant main effect on magnitude estimates, $F(2,32)=19.85, p<.0001$. That effect is shown in Figure 4. Higher mirror reflectivity leads to shorter distance estimates. Note that the vertical axis of Figure 4 has the same scale as that of Figure 3 to facilitate the visual comparison of the relative magnitudes of the effects of actual distance and reflectivity. However, this comparison is somewhat arbitrary because the overall sizes of the effects of both independent variables depend on the ranges tested. A more meaningful scaling of the effect of mirror reflectivity can be obtained by assuming that the effect of log reflectivity on magnitude estimates is linear (as is approximately the case in Figure 4). The best straight-line fit for Figure 4 yields a slope of -9.74 units of averaged magnitude estimates per $\log _{10}$ unit of mirror reflectivity. Combining this with the estimate of the slope in Figure 3 indicates that, within the ranges tested, decreasing mirror reflectivity by $1.0 \log _{10}$ unit has the same effect on distance estimates as an increase in actual distance of $3.44 \mathrm{~m}$.

The interaction of mirror reflectivity and actual distance was significant, $F(10,160)=2.36, p=.012$, and is illustrated in Figure 5. Although the effect of reflectivity is present at all distances, and is always in the direction of greater estimates with lower reflectivity, it appears to be stronger at shorter actual distances.

The two between-subjects variables, age and sex, each had a significant interaction with actual distance. The interaction of age and distance, $F(5,80)=3.09, p=.013$, is shown in Figure 6; the interaction of sex and distance, $F(5,80)=3.77, p=.0041$ is shown in Figure 7. Older subjects and male subjects consistently used smaller ranges of numbers in generating their estimates than did younger subjects and female subjects.

The effect of actual distance on estimates changed over the course of the four blocks that made up each subject's session. The interaction of distance and block, $F(15,240)=2.36, p=.0034$, is shown in Figure 8. Over time, subjects' estimates consistently became lower, but only for the longer distances.

Block also interacted significantly with sex, $F(3,48)=6.53, p=.0009$, as shown in Figure 9. Male subjects, more than female subjects, tended to use lower numbers later in the session. 


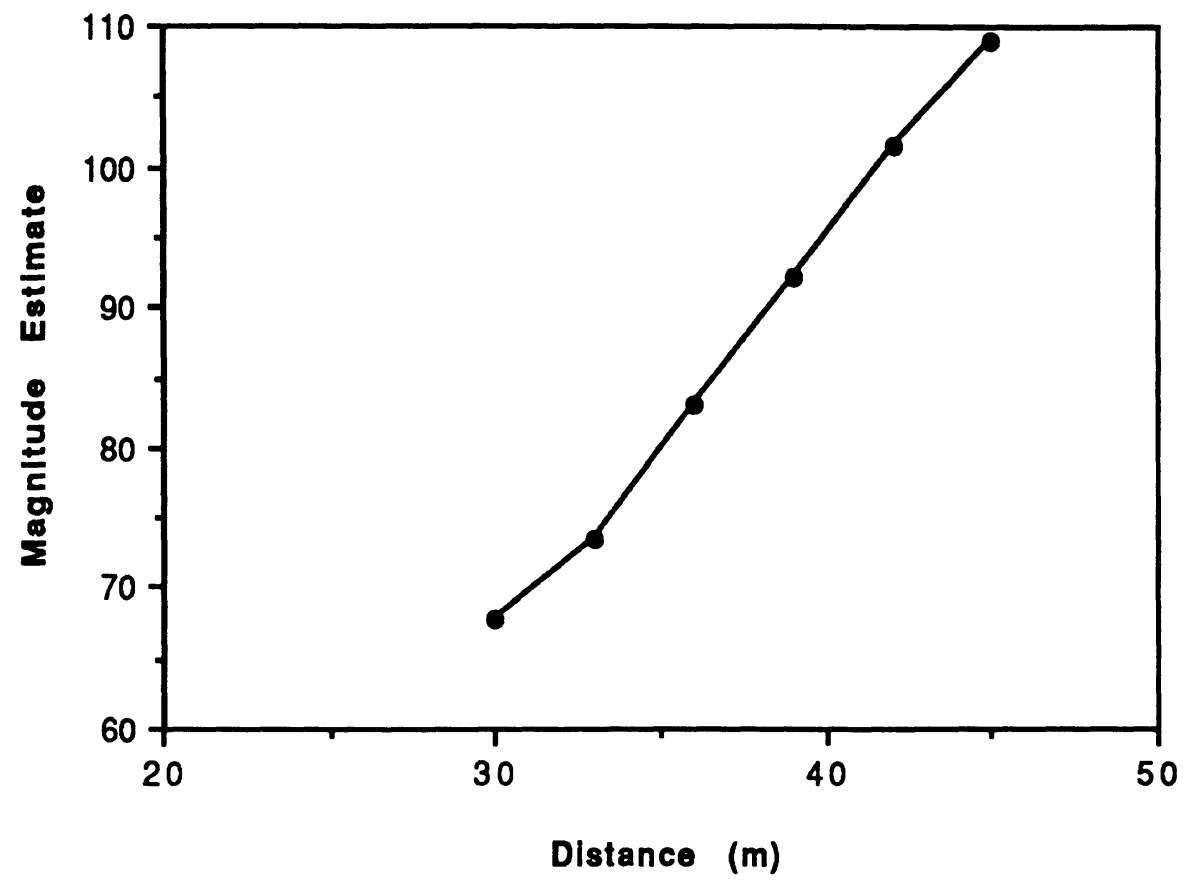

Figure 3. The main effect of actual distance on magnitude estimates of distance.

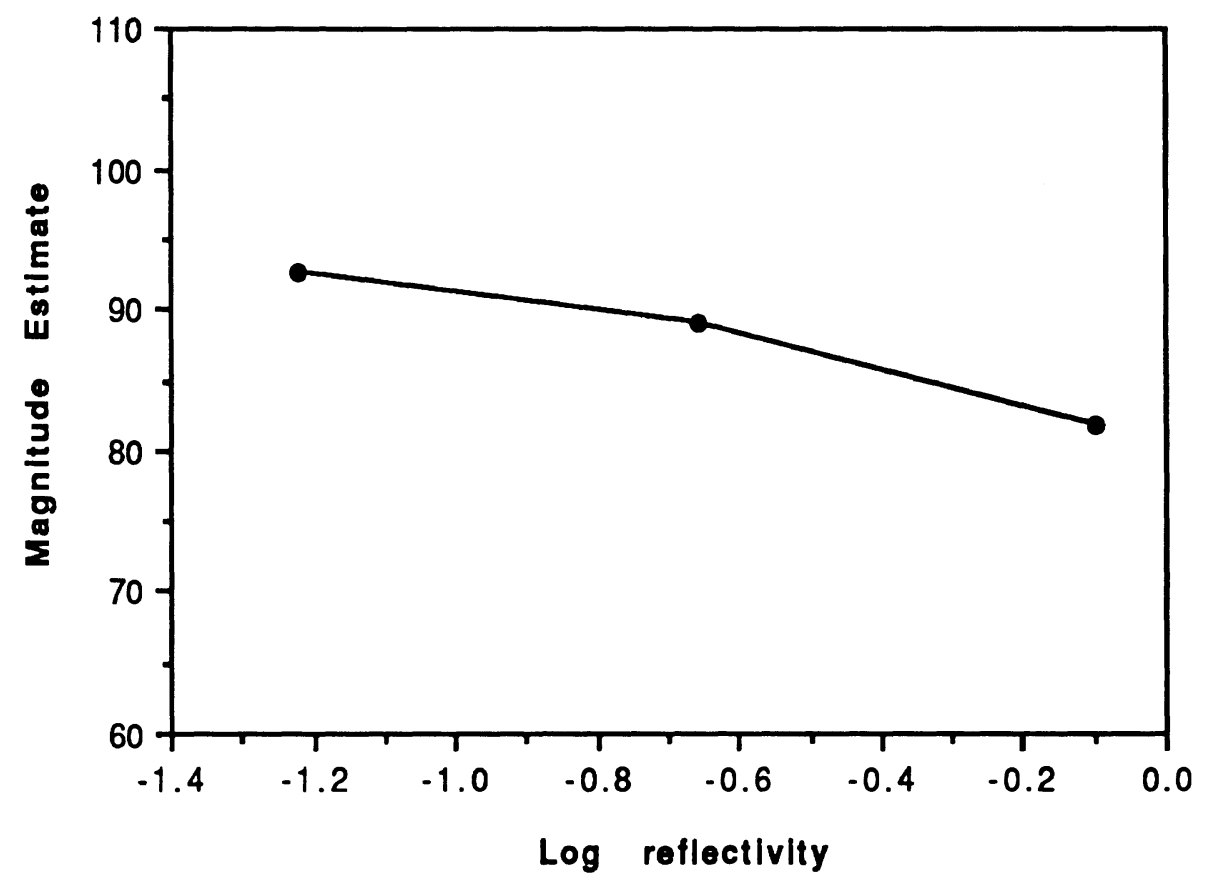

Figure 4. The main effect of $\log _{10}$ reflectivity of the rearview mirror on magnitude estimates of distance. Note that the vertical axis has the same scale as that in Figure 3, to facilitate comparison of the magnitude of the reflectivity effect to the effect of actual distance over the 15-m range tested. 


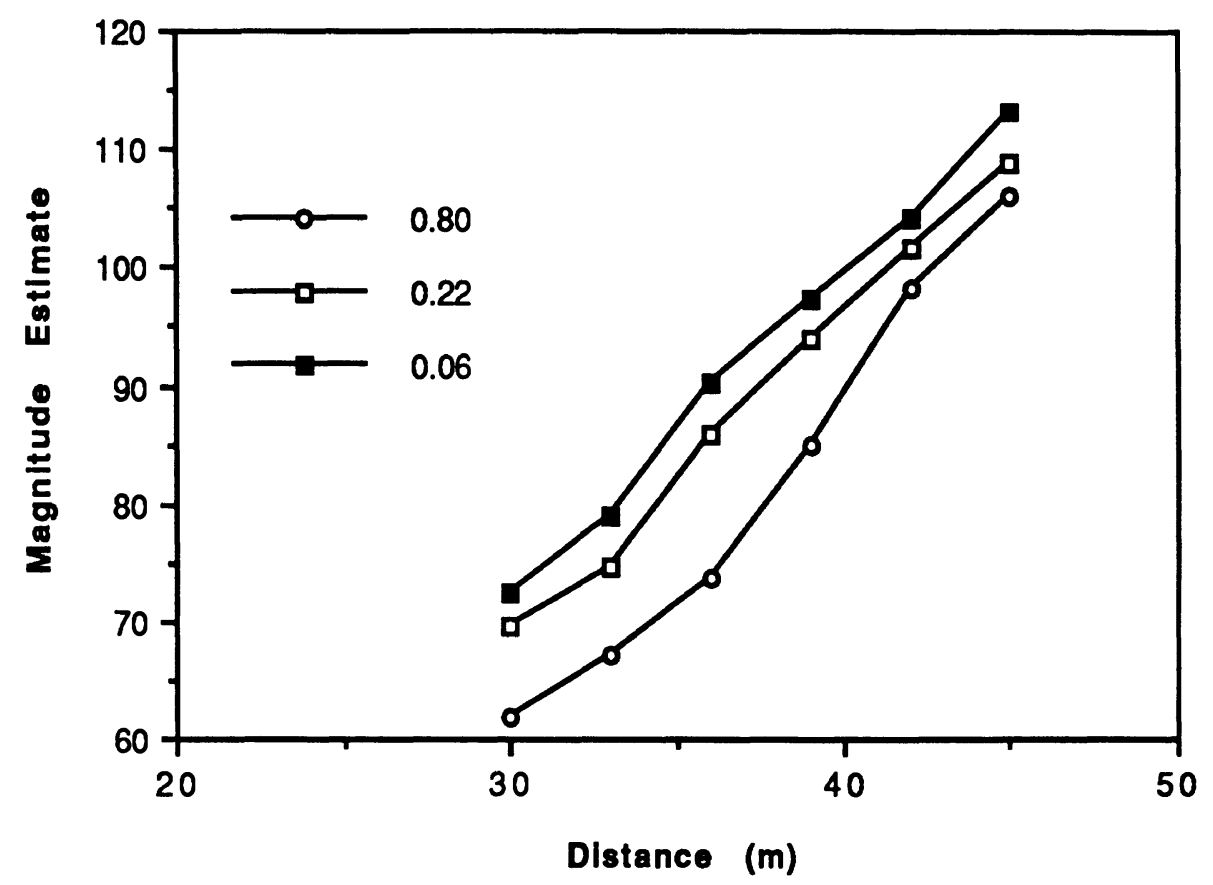

Figure 5. The effect of actual distance on magnitude estimates of distance for each level of mirror reflectivity.

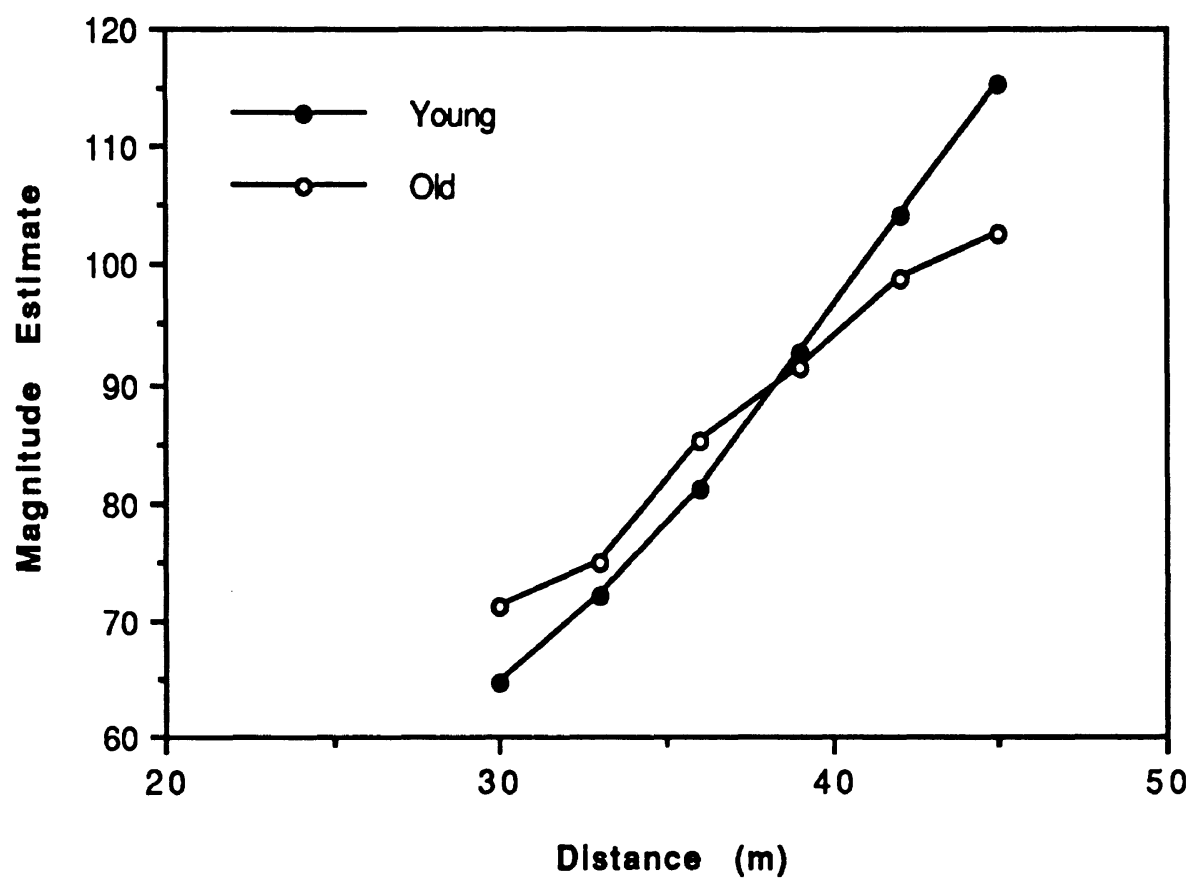

Figure 6. The effect of actual distance on magnitude estimates of distance for the younger and older groups of subjects. 


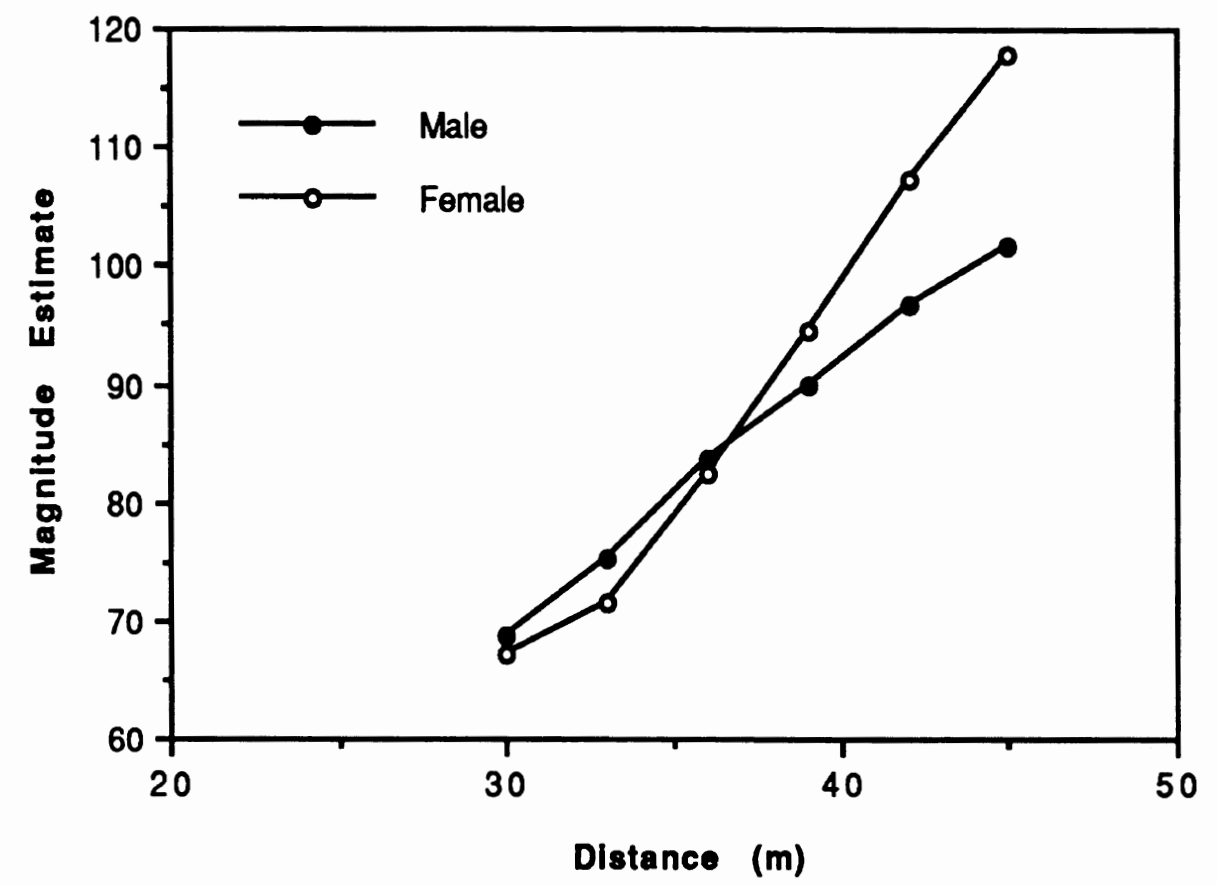

Figure 7. The effect of actual distance on magnitude estimates of distance for male and female groups of subjects.

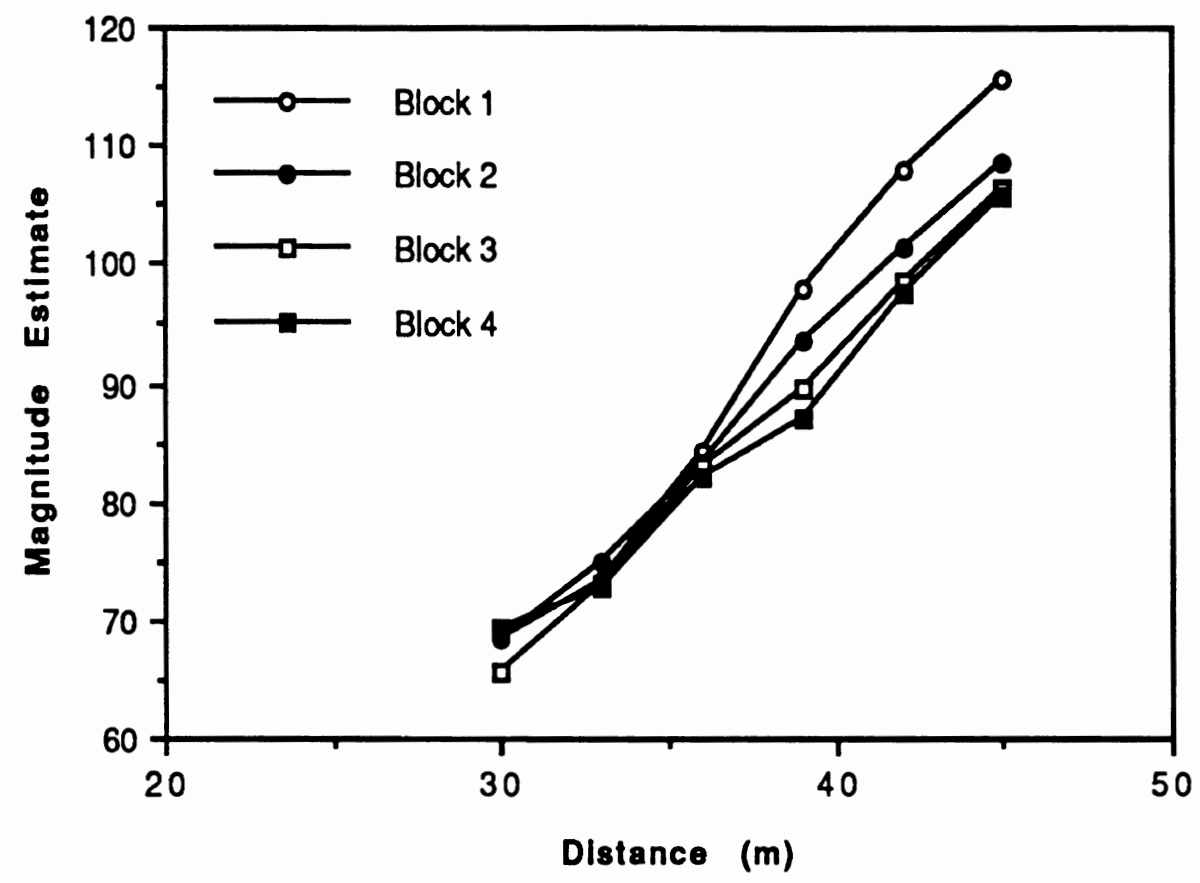

Figure 8. The effect of actual distance on magnitude estimates of distance for each block of trials within sessions. 
Effects on variability of estimates. Although effects of mirror reflectivity on the central tendency of judgments about distance are of potential importance, it can be argued that effects on the consistency of those judgments are even more important. Effects on central tendency can be regarded as miscalibrations, and it may be that people can become recalibrated with experience. But if judgments become more variable, then the transmission of information by the mirror has been degraded in a more fundamental way. Systematic distortions are always, at least in principle, reversible, but adding random noise is not.

In order to assess the effects of mirror reflectivity on the consistency of subjects' magnitude estimates, we performed separate analyses of variance on each subject's data. The goal was to remove everything that we could reasonably judge to be a systematic effect from the data in order to get the cleanest look at subjects' levels of random error. We applied the same model to each subject: an analysis of covariance that included mirror reflectivity and actual distance as factors. Because at least some subjects showed changes in their estimates over the course of blocks, as indicated in Figure 9, we included block as the covariate. Specifically, the model included 2 degrees of freedom for the main effect of reflectivity, 5 degrees of freedom for the main effect of distance, 10 degrees of freedom for their interaction, and 1 degree of freedom for the linear effect of block. Each of these elements appeared to be capturing systematic variation for a substantial number of subjects.

We used the residuals from these analyses to estimate random error. We computed the standard deviation (adjusted for the slight restriction due to taking out the linear effect of block) of residuals for each combination of reflectivity, distance, and subject. We then submitted these values to an analysis of variance including all subjects, with age and sex as betweensubjects factors and reflectivity and distance as within-subject factors. The analysis showed no significant effects. Because it is of special interest, the nonsignificant trend for the effect of reflectivity, $F(2,32)=0.131$, is illustrated in Figure 10 . Note that zero is included on the vertical axis because it is a meaningful baseline for comparing magnitudes in this case. There is very little evidence for an effect of mirror reflectivity.

In some ways the lack of significant effects is not surprising; variability is notoriously difficult to measure. Because of this, the standard cautions about accepting the null hypothesis are particularly applicable here. No set of data can prove that a difference is exactly zero; a null result merely puts an upper bound on how big a particular difference can plausibly be, and confidence intervals provide a way of quantifying that bound. We constructed a confidence interval for the possible effect of reflectivity on our standard deviation measure by computing the ratio of the standard deviation when the mirror was at its lowest reflectivity, to the standard deviation at the highest reflectivity. We did this for each subject and used the variability of the ratio over the group of 20 subjects to construct the confidence interval. The mean ratio over all 
subjects was 1.01, and the 90-percent confidence interval ranged from 0.93 to 1.09 . This means that if there is any change in the standard deviation of subjects' magnitude estimates due to mirror reflectivity over the range tested, it is unlikely to be by more than about 10 percent in either direction, relative to performance in the 0.80 reflectivity condition. 


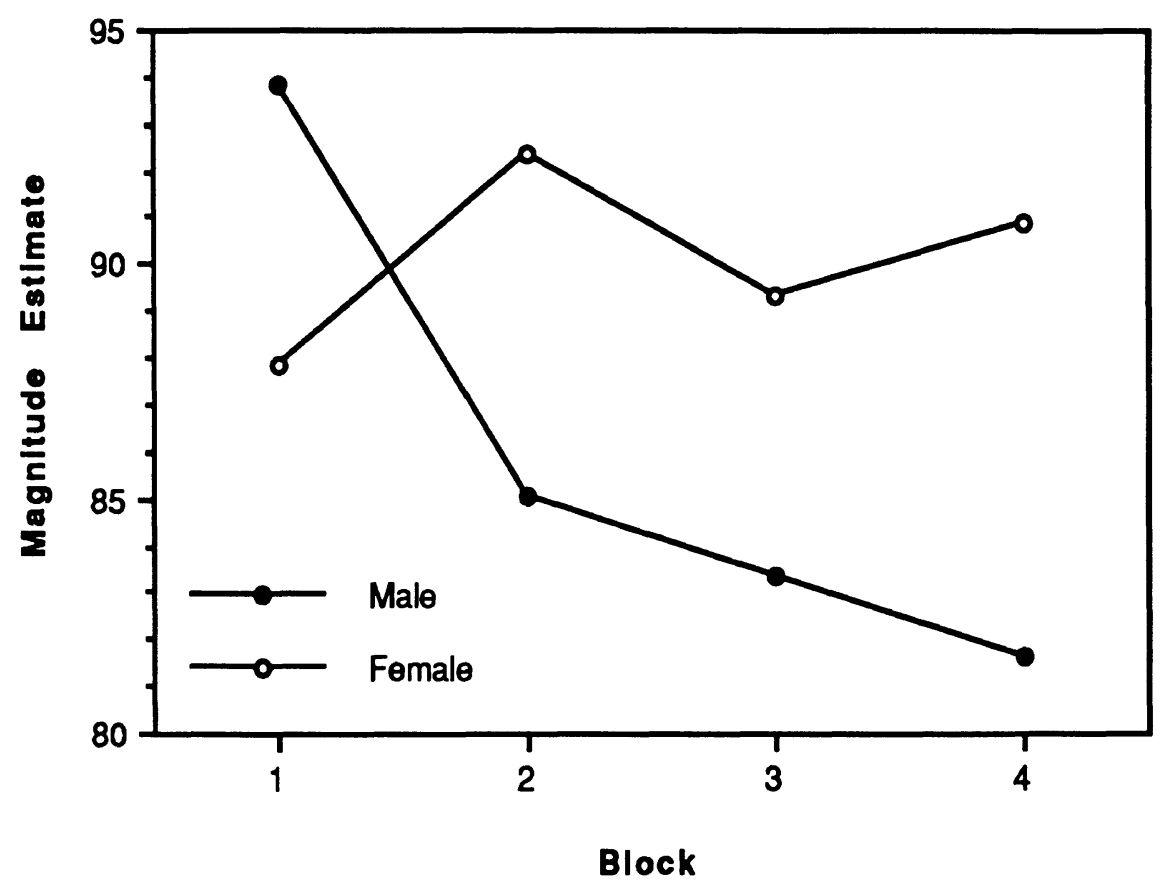

Figure 9. Magnitude estimates of distance by males and females over the course of the four blocks that made up the each subject's session.

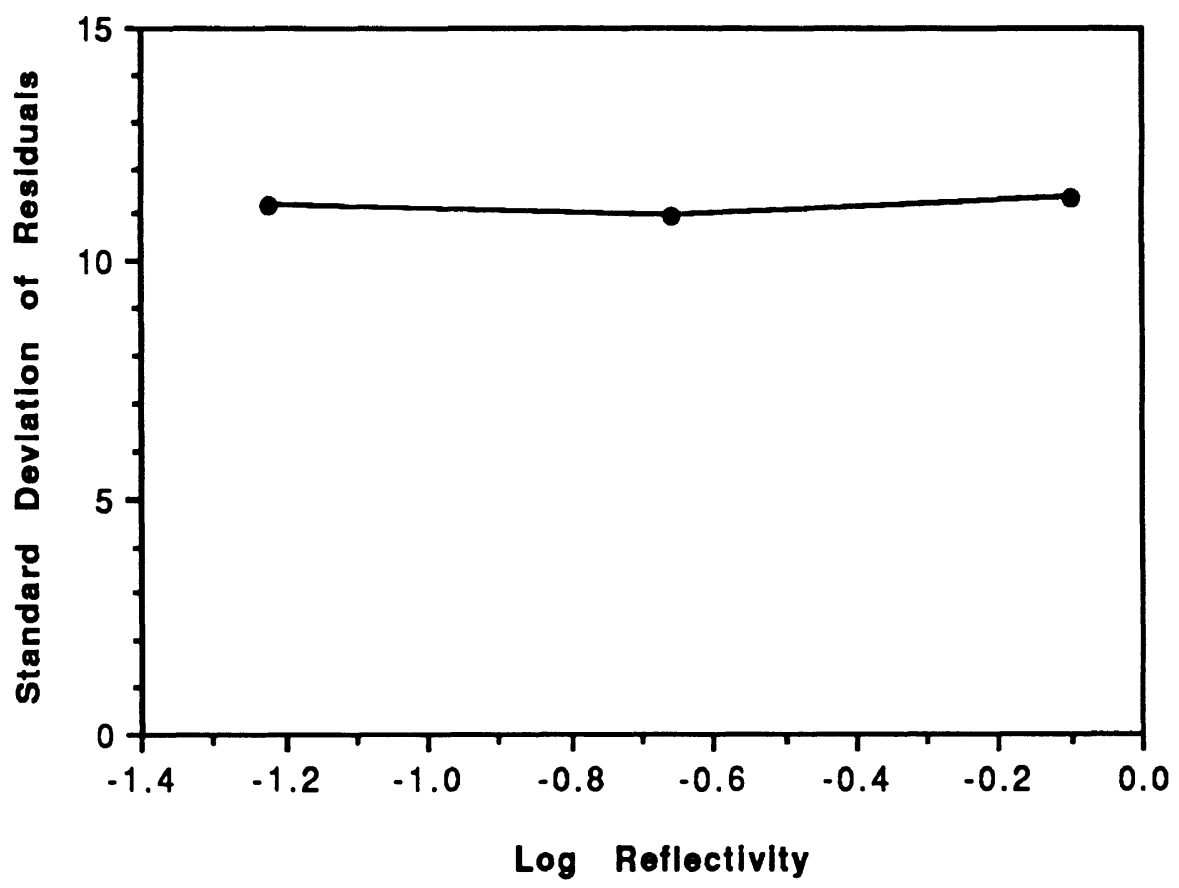

Figure 10. Standard deviation of residuals as a function of $\log _{10}$ mirror reflectivity. The residuals are from models applied to each subject individually. They include general effects of actual distance and mirror reflectivity, and a linear effect of time as represented by block. 


\section{Experiment 2: Distance Discrimination}

The results of Experiment 1 suggest that, although changes in rearview mirror reflectivity bias people's distance judgments, those changes do not affect the quality of the information available to them as measured by the consistency of their judgments. However, subjects in that experiment were not under time pressure. Perhaps a potential effect of mirror reflectivity was masked by strategies they were able to use when given the luxury of time. If so, performance in many of the demanding situations that occur in actual driving would certainly be different. The task used in Experiment 2 put subjects under strong time pressure, and also provided different and complementary ways of assessing the two aspects of performance that we discussed in connection with Experiment 1: central tendency and variability of distance perception. Subjects were allowed to view a stationary vehicle in a rearview mirror; the scene was briefly obscured; the vehicle was moved slightly nearer or farther away; and they were again allowed to view it. Their task was to decide as quickly as possible in which direction the vehicle had been moved. We measured reaction times and error rates.

\section{Method}

Subjects. Sixteen licensed drivers, eight in a younger group and eight in an older group, were paid to participate. The younger group ranged in age from 17 to 30 , with a mean age of 22.9; the older group ranged from 60 to 71, with a mean age of 64.2. Each age group consisted of four males and four females.

Apparatus. The same two vehicles used in Experiment 1 were used in this experiment. Because reaction time was an important measure in this experiment, a large shutter mechanism was added to the observer vehicle so that the view of the stimulus vehicle could be presented at a well-defined time. The shutter was constructed of a thin piece of plywood and positioned just behind the rear seat of the station wagon, at the front of the cargo area. When closed, the shutter completely blocked any view to the rear through the cabin of the vehicle. The shutter was hinged along the bottom edge, just below the top of the rear seat. It was spring loaded so that when an experimenter in the rear seat released a catch, the shutter would quickly snap open, rotating about the hinges on its lower edge into the cargo area. When the shutter came to rest against a cushioned stop in the cargo area, it closed a switch that started an electronic timer. The timer was also connected to a small panel, with two push buttons for indicating responses, that subjects could hold comfortably in their laps. When the subject pushed either button, the 
timer stopped and the elapsed time, along with a code for which button had been pushed, were recorded.

Field setup. Testing was conducted in the same parking lot as in Experiment 1. The observer vehicle was in the same position as before, and the stimulus vehicle could be placed in any of several positions behind it.

Stimuli. On each trial the subject was presented with the stimulus vehicle in two positions, first in a "base" position and then in a "displacement" position. There were two base positions, 30 and $38 \mathrm{~m}$ (measured, as in Experiment 1, between the centers of the front wheels of the vehicles). After a base position was presented, the stimulus vehicle was displaced by one of four distances: 8 meters nearer to the observer vehicle, 4 meters nearer, 4 meters farther, or 8 meters farther. Note that positions resulting from nearer displacements beginning at the far base position overlap the positions resulting from farther displacements beginning at the near base position. Consequently, there were seven total positions at which the vehicle could appear, as illustrated in Figure 11. The mirror reflectivities used were the same as in Experiment 1: $0.06,0.22$, and 0.80 . The stimulus vehicle was always presented with its lowbeam headlamps on.

Photometry. Illuminance at the center of the rearview mirror was measured as in Experiment 1. Measurements with the stimulus vehicle at each of the seven possible locations is shown in Figure 12. The location of the peak illuminance, at $34 \mathrm{~m}$, is consistent with the measurements taken for Experiment 1; however, the peak illuminance measured for Experiment 2 is lower. Because the same equipment and locations were used, we do not have an explanation for the difference other than error of measurement.

Design. There were two between-subjects variables: age and sex; and four withinsubject variables: base position of the stimulus vehicle, displacement of the stimulus vehicle, mirror reflectivity, and time (represented by dividing a session into thirds). Each subject served in a single session. Each of the 24 factorial combinations of base position (2 levels), displacement (4 levels), and mirror reflectivity (3 levels), was presented three times in each session, once during each of three blocks that made up the session. Within each of those blocks, mirror reflectivity was presented in three smaller blocks of eight trials each. The order of the three reflectivity blocks was randomized within each of the larger blocks, and the order of the base position and displacement combinations was randomized within each of the reflectivity blocks.

Procedure. Most aspects of the procedure were the same as in Experiment 1. Trials in Experiment 2 began with the stimulus vehicle at one of the two base positions. An experimenter in the back seat of the observer vehicle opened the shutter, allowing the subject to see the stimulus vehicle for about $5 \mathrm{~s}$. The shutter was then closed, and a second experimenter 
moved the stimulus vehicle to the appropriate displacement position. The car's headlamps were turned off just before it was moved, and turned back on when it was in the displacement position. This was done to reduce any information about the direction of the car's movement that the subject might be able to get from seeing movement of the headlamp beams on the scene in front of or beside the observer vehicle. The reappearance of the headlamps also served as an indicator to the experimenter in the observer vehicle that the stimulus vehicle was in position. That experimenter then reopened the shutter, and the subject indicated his or her decision about the direction of the stimulus car's displacement by pushing one of the two buttons, marked "nearer" and "farther," on the response panel. The shutter remained open until the subject responded. The subject was not asked to judge the extent of the displacement, only the direction. The interval during which the shutter was closed to allow the stimulus vehicle to move was about $5 \mathrm{~s}$. Subjects were alerted by the experimenter in the observer vehicle just before each time the shutter was opened, and they were instructed to be looking at the rearview mirror when it opened. Subjects were encouraged to be both fast and accurate.

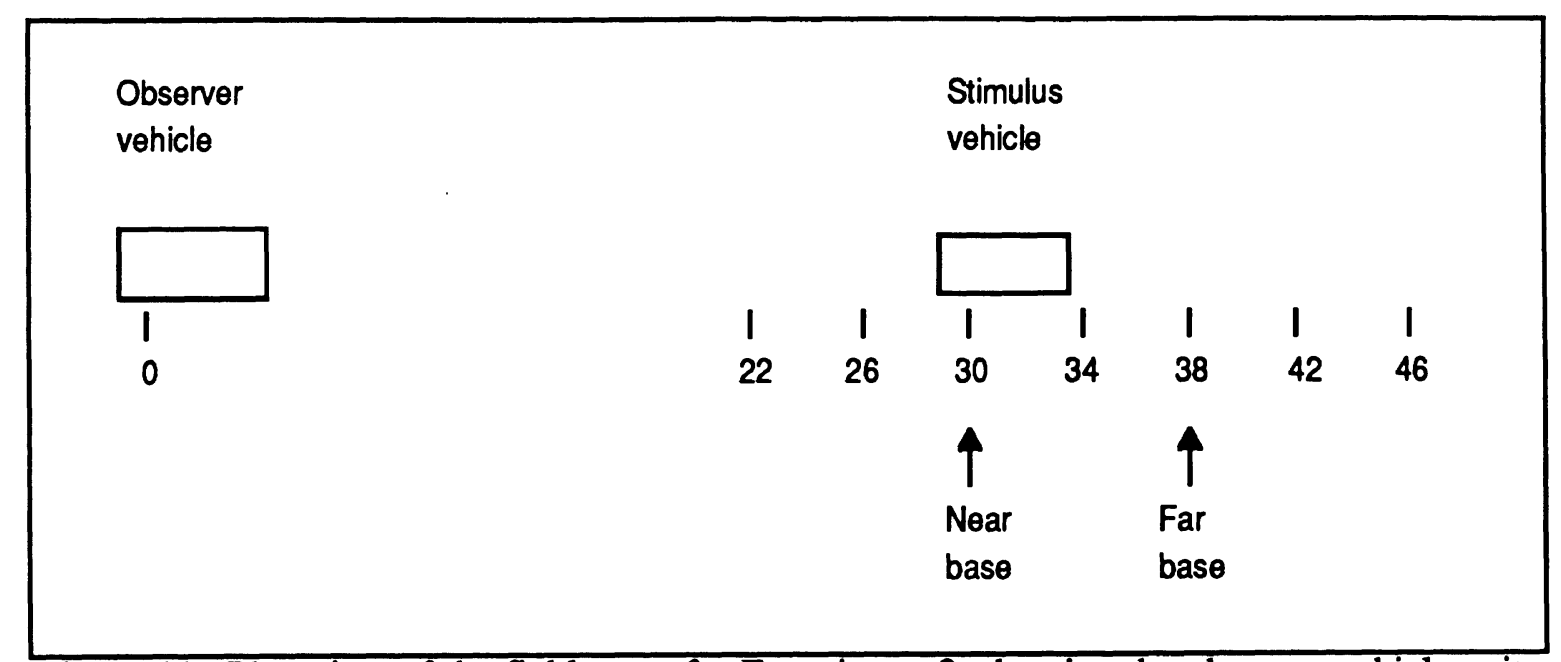

Figure 11. Plan view of the field setup for Experiment 2, showing the observer vehicle at its fixed position (front wheel centered at $0 \mathrm{~m}$ ) and the stimulus vehicle at the near base position (front wheel centered at $30 \mathrm{~m}$ ). 


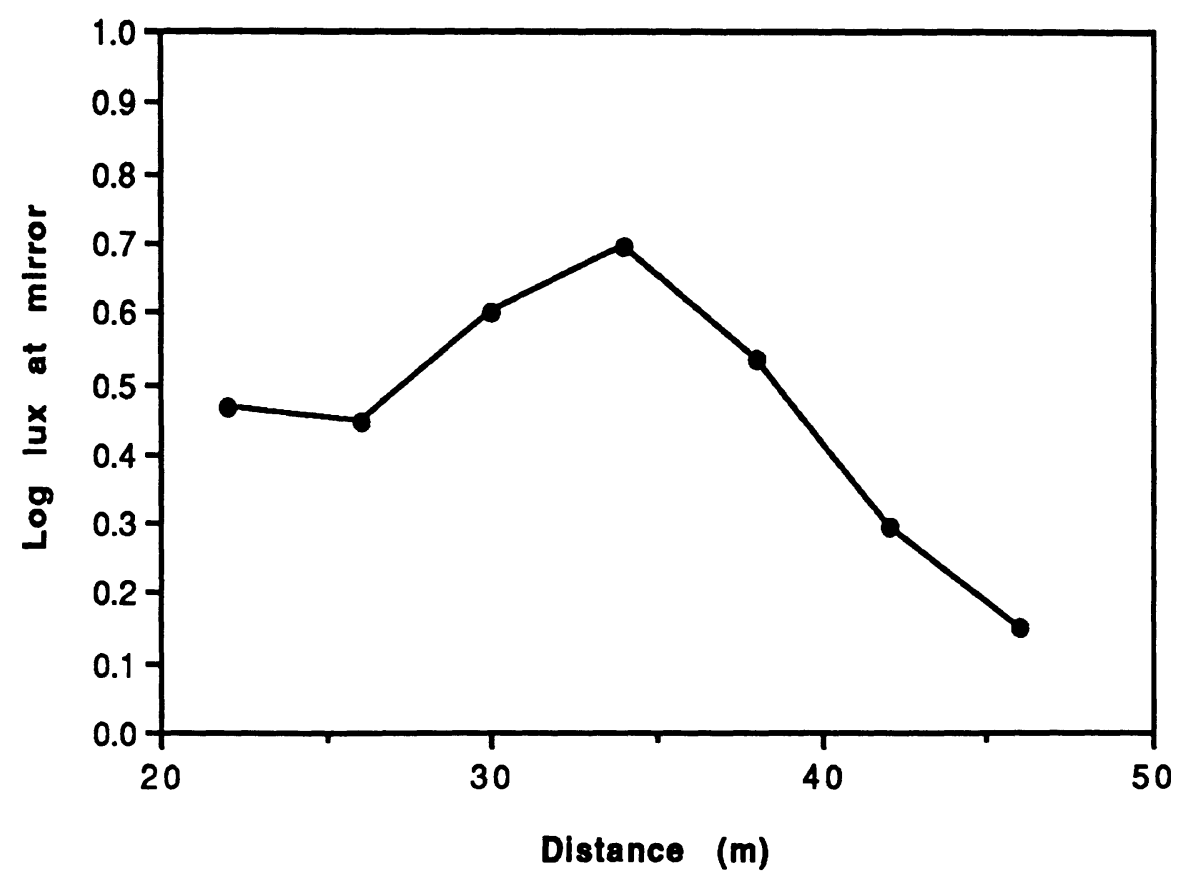

Figure 12. $\log _{10} \operatorname{lux}$ at the center of the rearview mirror, with the stimulus vehicle at each of the positions used in Experiment 2.

\section{Results and discussion}

Preliminary analyses of variance using sex, age, block, and reflectivity as factors indicated that there was a significant main effect of block on reaction time, $F(2,24)=5.41$, $p=.012$. Subjects became faster over the course of the sessions. Block did not have a significant effect on error rates, however, $F(2,24)=1.79, p=.19$. Both of these effects are shown in Figure 13. Block did not have a significant two-way interaction with mirror reflectivity for either reaction time, $F(4,48)=0.75, p=.56$, or error rate, $F(4,48)=1.39$, $p=.25$. The analysis for reaction time used data from correct trials only. In order to deal better with the problems of missing reaction-time data that resulted from errors, the data were collapsed across blocks for all further analyses.

Our main analyses were two parallel analyses of variance for reaction time and error rate. The reaction time data were from correct trials only. Each analysis included sex and age as between-subjects factors; and base position, displacement, and mirror reflectivity as withinsubject factors. Somewhat surprisingly, there were no significant main effects or interactions of sex or age on either of the dependent variables. None of those effects even approached significance. However, the nonsignificant trends in the data were reasonably in agreement with our expectations. Males were slightly faster (893 versus $1091 \mathrm{~ms}$ ) and more error-prone 
(.102 versus .073 error rate) than females. Older subjects were both slower (1097 versus 886 $\mathrm{ms})$ and more error-prone (.095 versus .080 error rate) than younger subjects.

Neither reaction time, $F(2,22)=1.07, p=.36$, nor error rate, $F(2,24)=0.14$, $p=0.87$, showed a significant main effect of mirror reflectivity. Because those potential effects are of special interest, the nonsignificant trends are shown in Figure 14. Over all 16 subjects, the mean difference between reaction time at the lowest reflectivity level and at the highest (lowest minus highest) was $-24 \mathrm{~ms}$. The 90 -percent confidence interval for this difference is from -84 to $59 \mathrm{~ms}$.

Both reaction time, $F(3,33)=9.41, p<.0001$, and error rate, $F(3,36)=13.28$, $p<.0001$, showed highly significant main effects of displacement, as shown in Figure 15. These effects are consistent with each other, longer reaction times are associated with higher error rates across the four different displacements. The pattern indicates that subjects find it easier to identify displacements when they are relatively large, and when they are toward, rather than away from, the observer position. It is not surprising that 8-m displacements are easier to identify than 4-m ones, but it is not as clear why the approaching displacements are more readily identified than the receding ones. One candidate explanation for the latter finding is that subjects are sensitive to proportional changes in visual angle between the headlamps. For the headlamp separation and the between-vehicle distances involved, the nonlinearity of visual angle with distance may be large enough to affect subjects' behavior. For example, at the $30-\mathrm{m}$ position the headlamps are separated by $2.33^{\circ}$. When they are brought nearer by $8 \mathrm{~m}$, that is increased to $3.17^{\circ}$, an increase of 36 percent over the original angle. When they are moved away by $8 \mathrm{~m}$ the angle is reduced to $1.84^{\circ}$, a reduction of only 21 percent below the original angle.

Both reaction time, $F(3,33)=3.10, p=.04$, and error rate, $F(3,36)=8.74$, $p=.0002$, are also affected by the interaction of displacement and base position. These effects are illustrated in the top and bottom panels of Figure 16, respectively. For both dependent variables, much of the pattern of the interaction seems to be captured by the pattern of main effects that is evident in Figure 15 being intensified at the near base position. That is consistent with the candidate explanation, in terms of proportional changes in visual angle, offered above for the apparent influence of the direction of displacement.

One additional two-way interaction, displacement by mirror reflectivity, was significant in the analysis of error rate, $F(6,72)=2.84, p=.015$, but not in the analysis of reaction times, $F(6,66)=1.66, p=.15$. The pattern of the interaction for error rate is shown in Figure 17. Most of the interaction appears to involve the smaller displacements. The error rates for the larger displacements (represented by the dotted lines in the figure) are uniformly 
low, perhaps low enough that any potential differences among them are obscured by a floor effect.

The part of the interaction involving the smaller displacements has a particularly interesting pattern, almost a perfect crossover. Consistent with the lack of a main effect of reflectivity on error rates, reflectivity does not affect the overall number of errors, but it has a marked effect on the balance of two kinds of errors. When reflectivity is high, subjects seldom make errors on trials with 4-m, nearer displacements (which would involve misidentifying them as farther), but they often make errors on trials with 4-m, farther displacements (misidentifying them as nearer). This pattern is weakened at the intermediate level of reflectivity, and reversed at the lowest level. When the mirror is most reflective, subjects more often judge the displacement to be nearer, when the mirror is least reflective, they more often judge the displacement to be farther. Although mirror reflectivity does not seem to affect the overall difficulty of the task, as indicated by the lack of main effects on reaction time or errors, it does seem to change subjects' bias to judge displacements as nearer or farther. To highlight these patterns, we have replotted, in Figure 18, the data shown in Figure 17 for the smalldisplacement conditions. Figure 18 shows the overall error rate and the proportion of displacements judged as farther as functions of $\log _{10}$ reflectivity.

It is not immediately clear why reflectivity should affect subjects' tendencies to judge displacements as being in one direction or another. Subjects were to judge the position of the vehicle in the second presentation of a trial against the position that they had just seen in the first presentation, seconds earlier. During a single trial, both presentations were always seen with the mirror at the same reflectivity level, and thus any effect of reflectivity on distance perception should cancel out of a relative judgment. This suggests that effects of reflectivity on perceived distance itself (if any) were irrelevant for determining the subjects' proportions of nearer and farther judgments, and that reflectivity had its effect in some other way, perhaps by changing their response biases. 


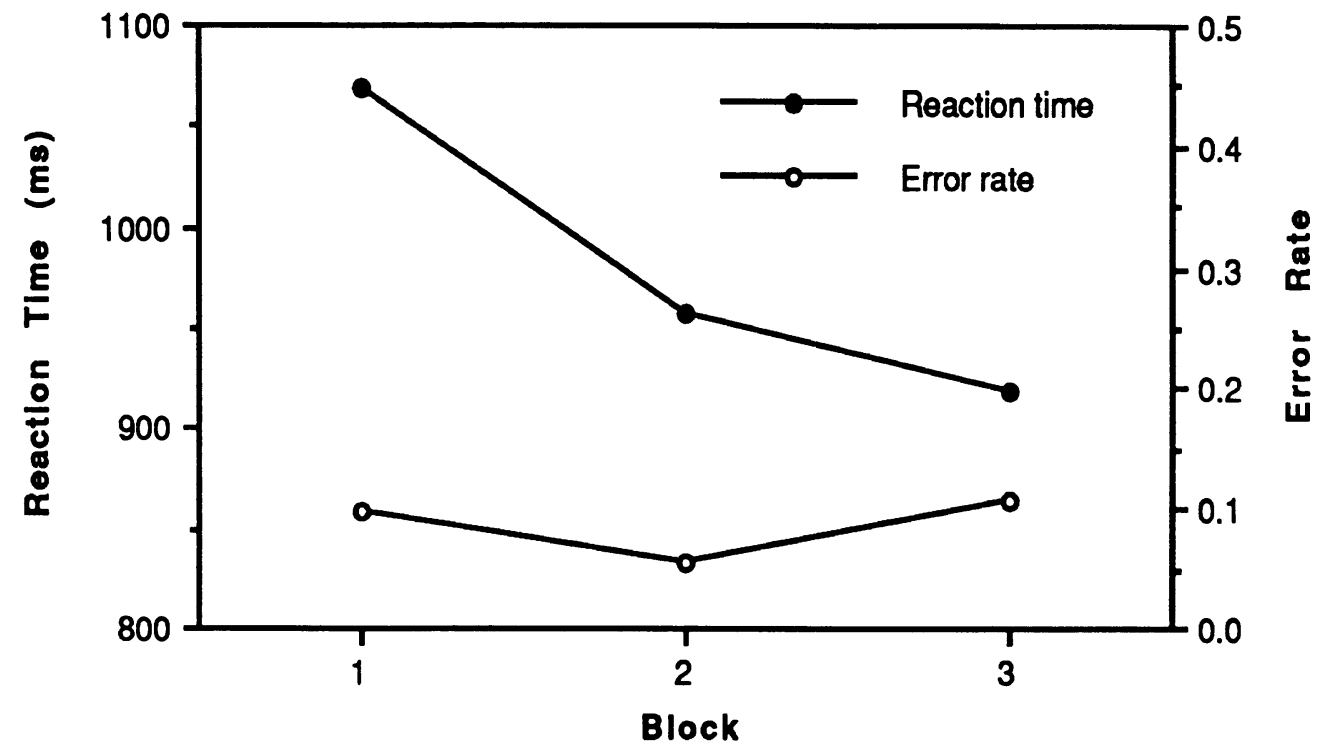

Figure 13. Reaction time (which shows a statistically significant effect) and error rate (which does not) as functions of block.

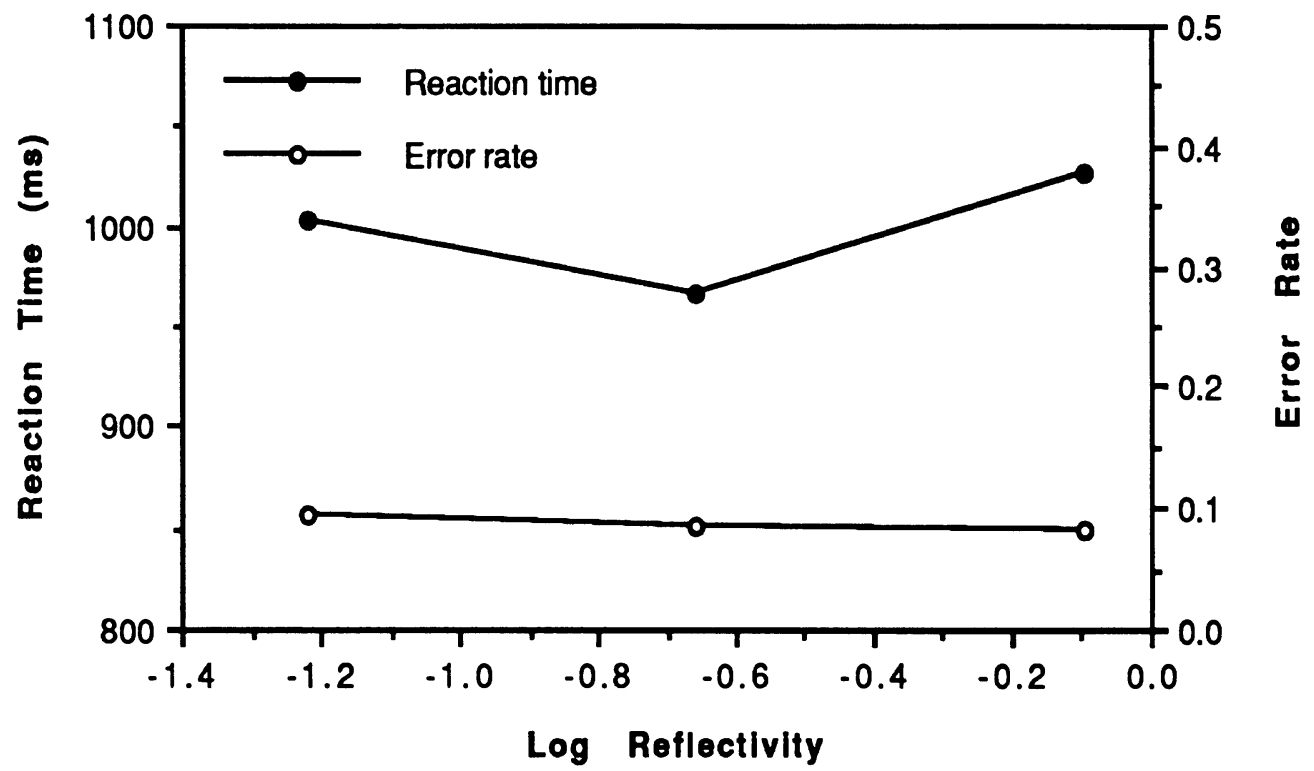

Figure 14. Reaction time and error rate as functions of reflectivity. Neither of these main effects approached significance. 


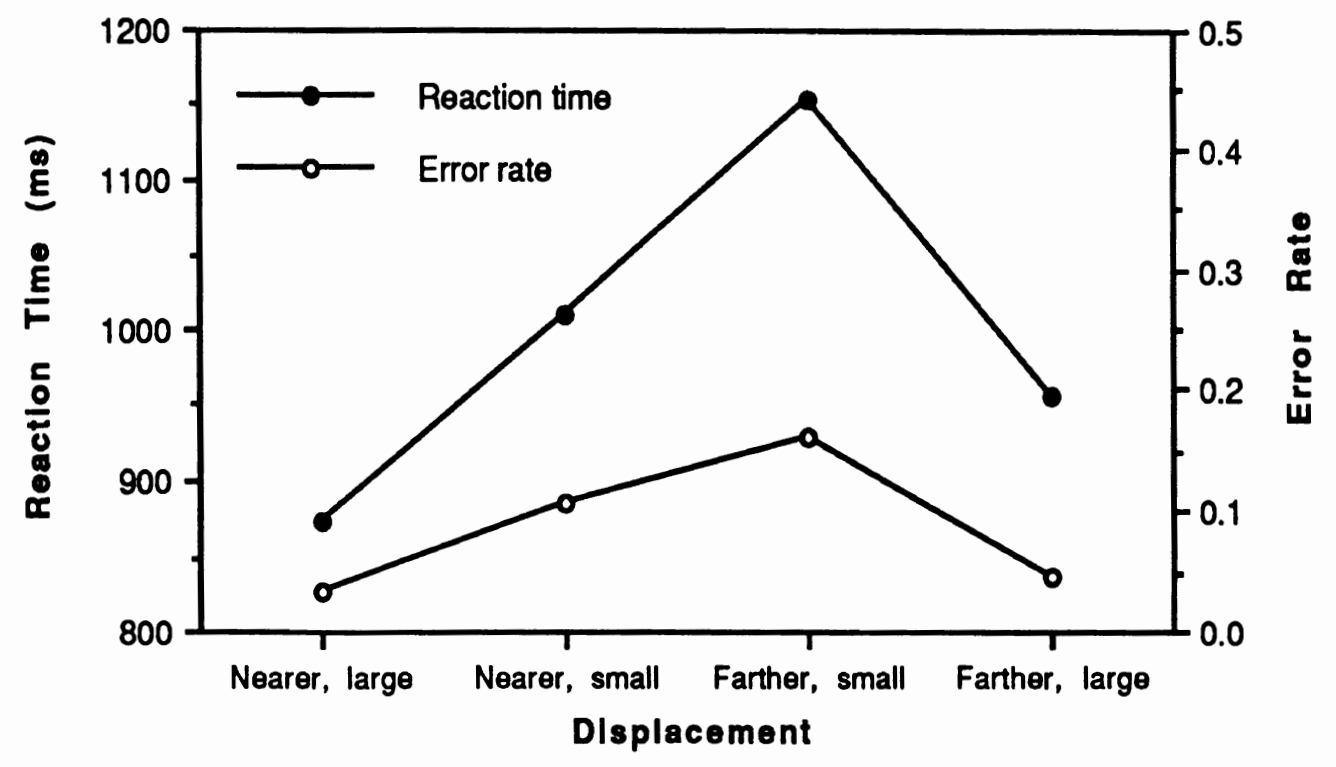

Figure 15. Reaction time and error rate as functions of displacement. Both effects are statistically significant and the patterns are similar. 

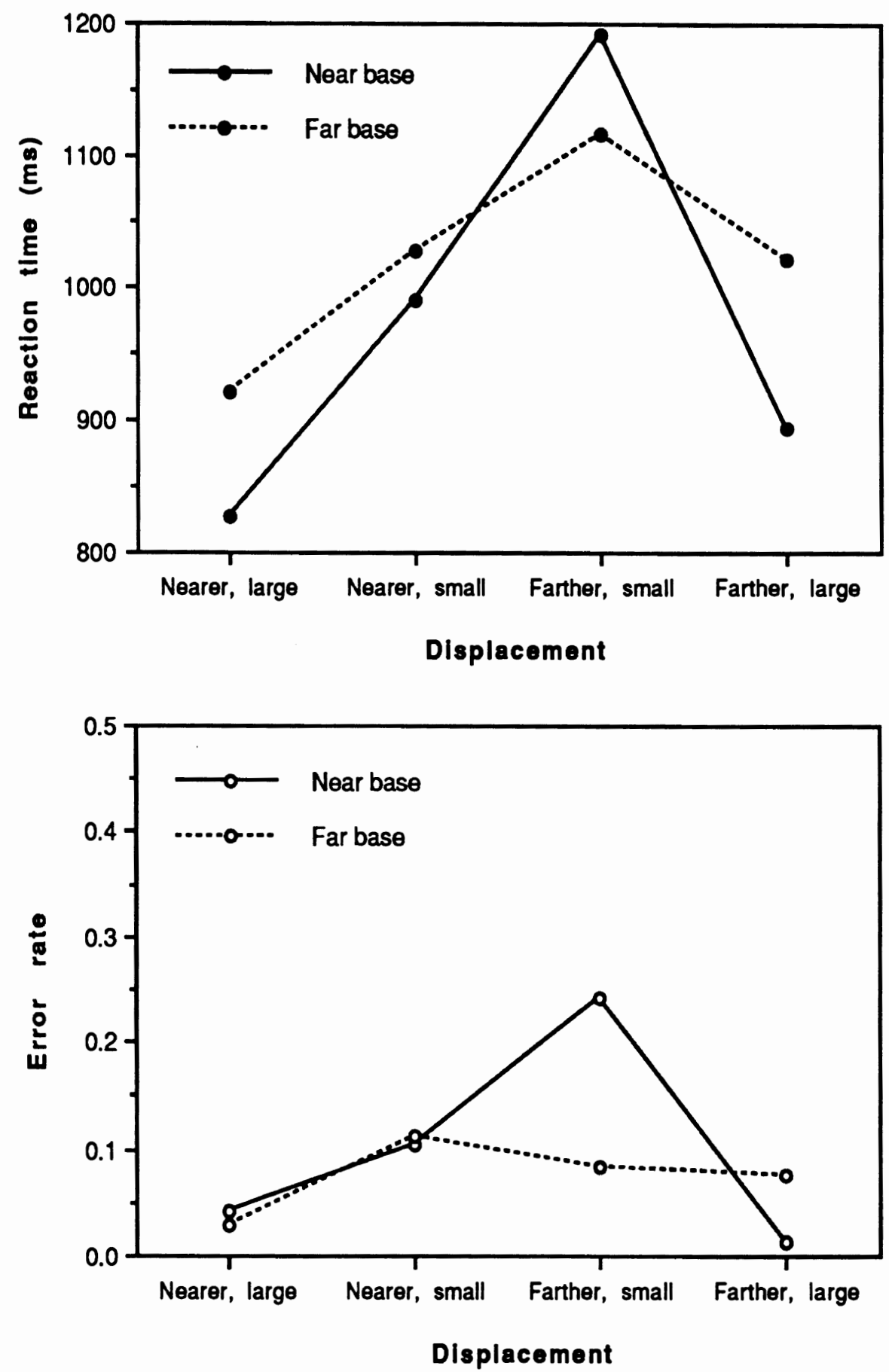

Figure 16. The interactions of displacement and base position for reaction time (upper panel) and error rate (lower panel). Both interactions are statistically significant. 


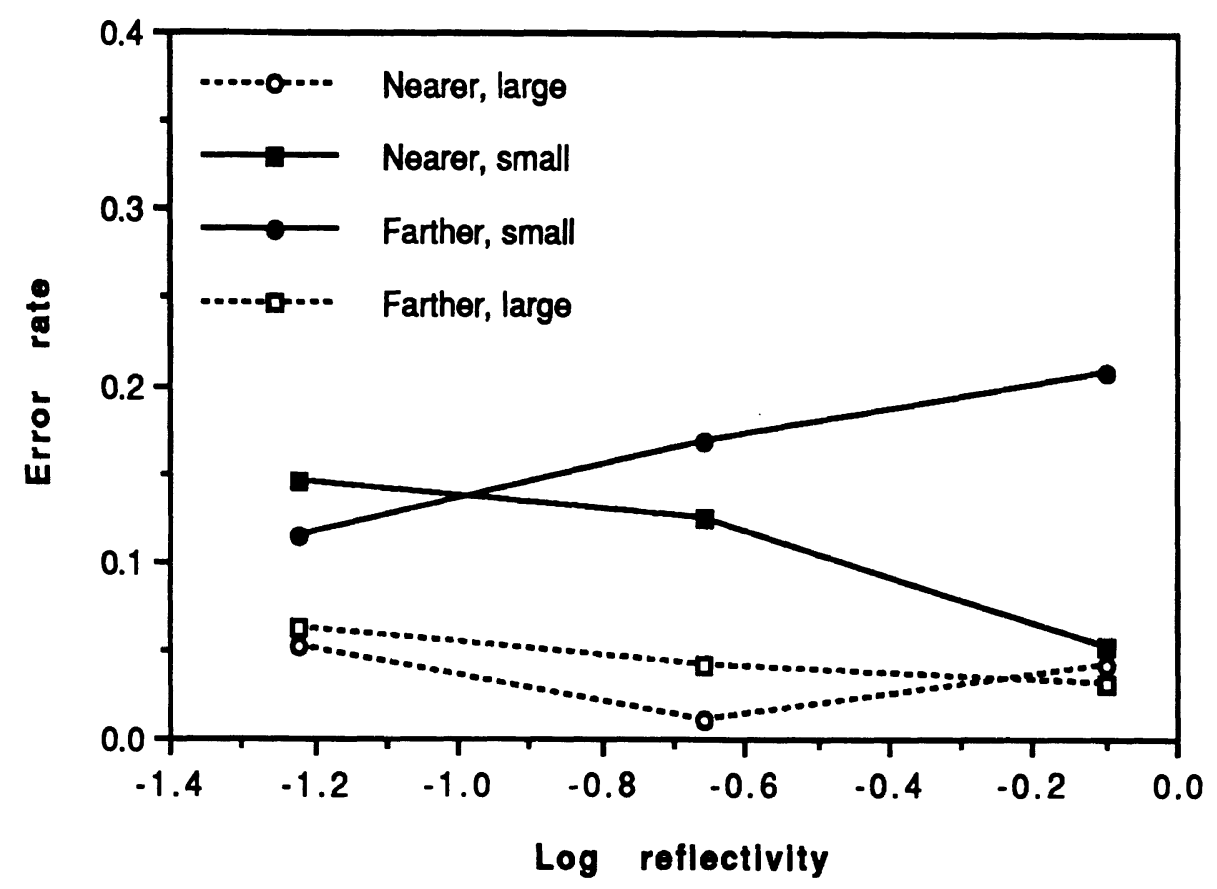

Figure 17. The interaction of displacement and mirror reflectivity for error rate. The effect is statistically significant, and appears to be due largely to a cross over involving data from the smaller displacements.

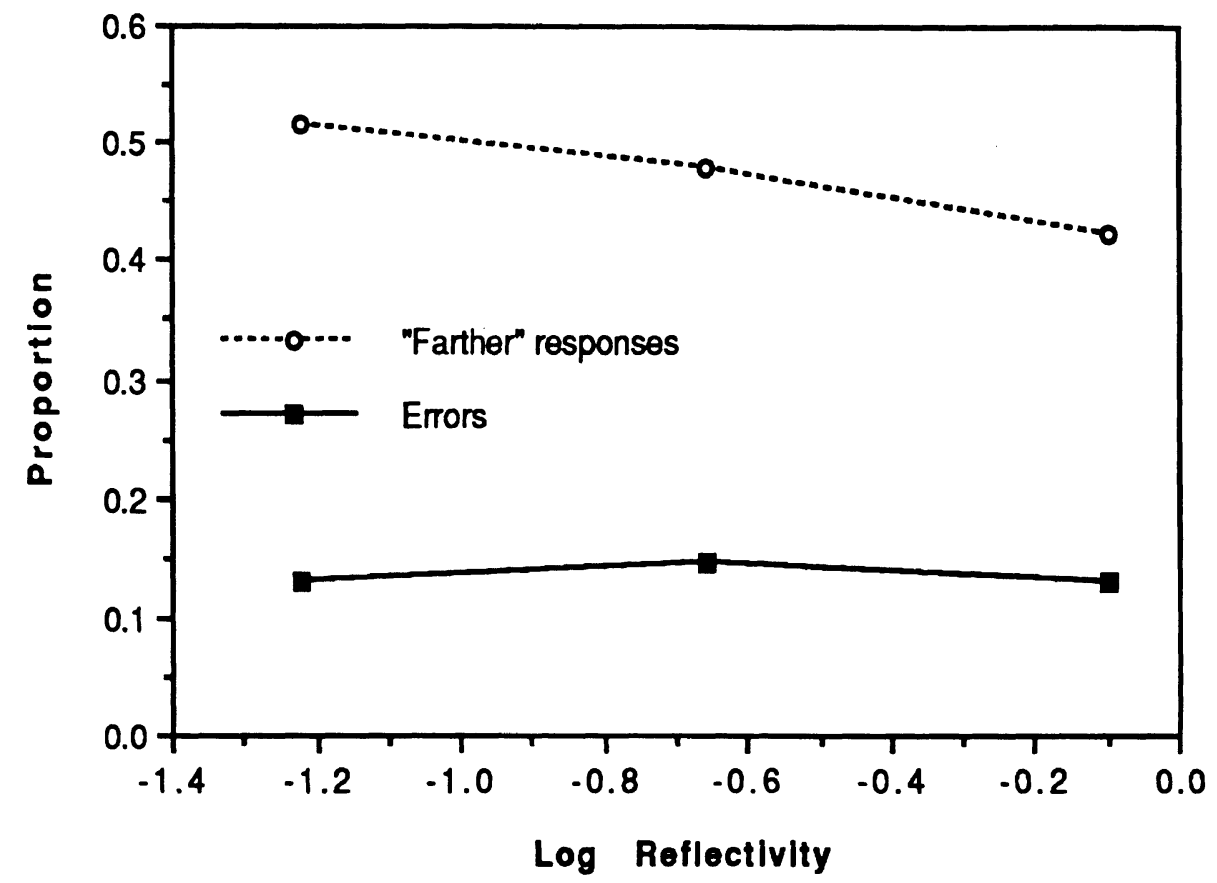

Figure 18. Proportions of "farther" responses and total errors of both types as a functions of $\log _{10}$ mirror reflectivity, from the small-displacement conditions. These graph is a simple replotting of the data for the small-displacement conditions in Figure 17. 


\section{General Discussion}

The results of these two experiments are consistent. Both experiments showed virtually null effects of rearview mirror reflectivity on variable errors in distance judgment. As with all null results, these data should not be interpreted as proving that there is absolutely no effect of reflectivity, but rather as putting upper limits on how large any potential differences may be. Those limits can be quantified by the confidence intervals for the effects of reflectivity on variability of magnitude estimates (Experiment 1) and on reaction time (Experiment 2).

Experiment 1 showed clear evidence for an effect of rearview mirror reflectivity on the constant error of distance estimates, with distance estimates being greater when reflectivity was lower. It is not straightforward to compare the results of Experiment 1 and 2 in terms of their implications for constant error. The tasks used were rather different. For the comparative judgments used in Experiment 2, any constant error due to reflectivity should have simply canceled out, making assessment of constant error impossible. Nevertheless, subjects in Experiment 2 showed a response bias to judge that stimuli were farther away as reflectivity decreased.

The relative importance of variable and constant errors for distance judgments is open to debate. However, as we mentioned earlier, constant errors may be more amenable to correction. Constant errors may be relatively easily corrected by recalibration resulting from general experience or specific training. Increased variable error resulting from the addition of random noise is more likely to be irreversible. Constant error is determined more by what the perceiver decides to do with the information available; variable error is more determined by the quality of the information itself.

\section{Implications for optimal reflectivity}

The results for variability of magnitude estimates, reaction time for distance discrimination, and accuracy of distance discrimination all suggest that quality of distance information does not vary with mirror reflectivity, and, consequently, that quality of distance information is not an important factor in determining optimal mirror reflectivity. This conclusion is consistent with the view outlined in the Introduction, that, because moving vehicles are marked by highly luminous lamps, those vehicles can be seen adequately over a wide range of mirror reflectivity. However, several limitations of the present results should be highlighted. The demands that subjects in these experiments faced were not as heavy as in actual driving. In these experiments the rearward field of view was simple; there was always one, and only one, vehicle visible in the rearview mirror. Also, the subjects did not have to 
perform additional tasks that are present in actual driving, such as maintaining lane position and avoiding obstacles in the forward field.

The results for constant error from Experiment 1 are empirically very clear. However, their implications are limited by two major concerns: (1) the question of how important constant error is in light of the possibility of recalibration with experience, and (2) the question of whether the effects observed here for magnitude estimation would generalize to other tasks, such as lane-change decisions. Nevertheless, the constant error results are potentially important, and appear to merit further investigation.

\section{References}

Flannagan, M. J., \& Sivak, M. (1990). Nighttime effectiveness of rearview mirrors: Driver attitudes and behaviors. In Vehicle lighting and driver visibility for the 1990s, SP-813 (pp. 69-79). Warrendale, PA: Society of Automotive Engineers.

Lynam, N. R. (1987). Electrochromic automotive day/night mirrors (SAE Technical Paper Series No. 870636). Warrendale, Pennsylvania: Society of Automotive Engineers.

Mansour, T. M. (1971). Driver evaluation study of rear view mirror reflectance levels (SAE Technical Paper Series No. 710542). New York: Society of Automotive Engineers.

Marks, L. E. (1974). Sensory processes: The new psychophysics. New York: Academic Press.

Olson, P. L., Jorgeson, C. M., \& Mortimer, R. G. (1974). Effects of rearview mirror reflectivity on drivers' comfort and performance (Report No. UM-HSRI-HF-74-22). Ann Arbor, MI: The University of Michigan Highway Safety Research Institute.

Stevens, S. S., \& Galanter, E. H. (1957). Ratio scales and category scales for a dozen perceptual continua. Journal of Experimental Psychology, 54, 377-411.

Ueno, H., \& Otsuka, Y. (1988). Development of liquid crystal day and night mirror for automobiles (SAE Technical Paper Series No. 880053). Warrendale, Pennsylvania: Society of Automotive Engineers. 